Edinburgh 2002/03

LAPTH-904/02

March 2002

\title{
A next-to-leading order study of photon-pion and pion pair hadro-production in the light of the Higgs boson search at the LHC.
}

\author{
T. Binoth ${ }^{b}$, J. Ph. Guillet ${ }^{a}$, E. Pilon ${ }^{a}$ and M. Werlen ${ }^{a}$ \\ a Laboratoire d'Annecy-Le-Vieux de Physique Théorique LAPTH, \\ Chemin de Bellevue, B.P. 110, F-74941 Annecy-le-Vieux, France \\ ${ }^{b}$ Department of Physics and Astronomy, University of Edinburgh, \\ Edinburgh EH9 3JZ, Scotland, UK
}

\begin{abstract}
We discuss the production of $\gamma \pi^{0}$ and $\pi^{0} \pi^{0}$ pairs with a large invariant mass at collider energies. We present a study based on a perturbative QCD calculation at full next-to-leading order accuracy, implemented in the computer programme DIPHOX. We give estimations for various observables, which concern the reducible background to the Higgs boson search in the channel $H \rightarrow \gamma \gamma$, in the mass range $80-140 \mathrm{GeV}$ at the LHC. We critically discuss the reliability of these estimates due to our imperfect knowledge of fragmentation functions at high $z$ and a subtle interplay between higher order corrections and realistic experimental cuts. We conclude that, whereas the invariant mass spectrum of photon-pion pairs is theoretically better under control, in the dipion case large uncertainties remain.
\end{abstract}




\section{Introduction}

A major motivation for the CERN Large Hadron Collider (LHC) experiments [1-4] is to shed light into the mechanism of electroweak symmetry breaking. As the LEP precision measurements favour a relatively light Higgs boson, its decay into a pair of photons is a prominent search channel which deserves intense consideration from the theoretical side. The inclusion of QCD radiative corrections is mandatory not only for the signal but also for the background if one is aiming towards reliable predictions. In this respect it is important to note that especially at LHC, but also at the Tevatron, prompt photon signals - photons which do not stem from mesonic decays - are in general contaminated by $\pi^{0}$ events. The photon pairs from the mesonic decay which are very collimated, if the pions have a high momentum compared to the pion mass, may appear as single (fake) photons inside the detector. This background is especially worrisome in collider experiments, such as the Collider Detector at Fermilab (CDF) [5] and D0 [6] experiments at the Fermilab Tevatron, where the pions cannot all be reconstructed event by event. At the LHC $\gamma \pi^{0}$ and $\pi^{0} \pi^{0}$ production represent an important fraction of the reducible background to the search of neutral Higgs bosons in the channel $H \rightarrow \gamma \gamma$ in the intermediate mass range $80-140 \mathrm{GeV}$. Since the jet-jet cross section is about eight orders of magnitude larger than the expected Higgs boson signal for a standard model Higgs boson, this reducible background is overwhelming before any selection cut is imposed.

Regarding both issues of prompt diphoton production and Higgs boson search, various cuts, especially isolation cuts in transverse energy have to be imposed to the candidate events, in order to reject most of this background. Yet, the actual fraction of this background which passes these cuts is not negligible, and it is not well known. Up to now its estimations rely on Monte Carlo simulations encoding the partonic subprocesses at the leading logarithmic (LL) accuracy only. We may thus worry about large higher order corrections which may be not accurately reproduced by parton showers. Furthermore, the hadronisation models matched with the partonic ingredients in these simulations are tuned to describe correctly the bulk of pion production, but they may not be accurate in the kinematical region relevant for the pions which pass the cut. A tentatively quantitative study of isolated $\gamma \pi^{0}$ and $\pi^{0} \pi^{0}$ production, confronted to a careful analysis of data collected at the Tevatron could improve this situation. The motivation of this article is to provide a study of full next-to-leading order (NLO) accuracy, based on the computer code DIPHOX as an attempt in this direction.

The computer program DIPHOX on which our study relies is a Monte Carlo code of partonic event generator type. This code has been designed to describe the production of pairs of particles in hadronic collisions, accounting for all contributing partonic processes at full NLO accuracy. These particles can be prompt photons or hadrons, in particular neutral pions. This code is flexible enough to accommodate various kinematic or calorimetric cuts. Especially, it allows to compute cross sections for isolated $\gamma \pi^{0}$ and $\pi^{0} \pi^{0}$ pairs, for any infrared and collinear safe isolation criterion which can be implemented at the partonic level. The physical content and schematical description of DIPHOX has been given in Ref. [7], where we have concentrated on the case of diphoton production [8-10]. In Ref. [11] we recalled briefly how to use this code also in the case of $\gamma \pi^{0}$ and $\pi^{0} \pi^{0}$ production, and we focused on the phenomenology of dipion production at fixed target energies, with a comparison to the data of the E706 experiment [12] at the Tevatron. The aim of this comparison was to confront the theoretical framework on which DIPHOX relies to existing dipion data in order to validate this framework before transporting this knowledge to collider energies [13] using the factorisation property of perturbative QCD.

The main goal of the present article is to provide a NLO prediction for the reducible background to Higgs boson searches in the mass range $80-140 \mathrm{GeV}$ at the LHC. In section 2 we present estimates for distributions which are of interest regarding the Higgs boson search, such as the distributions of invariant mass and transverse momentum of a pair. In section 3 we will extensively discuss the uncertainties of our next-to-leading order approach induced by severe experimental cuts. Finally 
section 4 gathers our conclusions and some outlook.

\section{Predictions for the $\gamma \pi^{0}, \pi^{0} \pi^{0}$ background at the $\mathbf{L H C}$}

In this section we discuss NLO predictions for the $\gamma \pi^{0}$ and $\pi^{0} \pi^{0}$ reducible background to the neutral Higgs boson search channel $H \rightarrow \gamma \gamma$ in the intermediate mass range $80-140 \mathrm{GeV}$ at the LHC. All NLO curves where computed with the DIPHOX code, version (1.2) ${ }^{1}$. For all figures we are using the MRST2 [14] parton distribution functions, and, if not stated differently, the KKP fragmentation functions [15]. For all plots given below the following cuts on the transverse momenta, pseudo rapidities of the photons/pions and their invariant mass were applied:

$$
\begin{aligned}
p_{T}\left(\gamma_{1}, \pi_{1}\right) & >40 \mathrm{GeV} \\
p_{T}\left(\gamma_{2}, \pi_{2}\right) & >25 \mathrm{GeV} \\
|y(\gamma, \pi)| & <2.5 \\
80 \mathrm{GeV} & <m_{\pi \pi, \gamma \pi, \gamma \gamma}<140 \mathrm{GeV} .
\end{aligned}
$$

In addition, isolation cuts are imposed to reject events which are accompanied by a certain amount of hadronic energy, as this is typically not the case for photonic Higgs decay. A photon is said to be isolated if, in a cone in rapidity and azimuthal angle about the photon direction, the amount of deposited hadronic transverse energy $E_{T}^{\text {had }}$ is smaller than some value $E_{T \max }$ fixed by the experiment:

$$
E_{T}^{h a d} \leq E_{T \max } \quad \text { inside } \quad\left(y-y_{\gamma}\right)^{2}+\left(\phi-\phi_{\gamma}\right)^{2} \leq R^{2}
$$

The actual experimental criteria, which are defined at the detector level, are much more complicated than the simple one given by Eq. (2), see [5,6]. They cannot be implemented in a partonic calculation. The schematical criterion of Eq. (2) is a modelization of their effects at the parton level. The topic of isolation of photons based on the above criterion is extensively discussed in the literature $[17-21,16]$. We stress that there is a subtle interplay between higher order corrections and the experimental cuts. These subtleties will be discussed in the next section.

In Fig. 1 we show the invariant mass spectra of $\gamma \gamma, \gamma \pi^{0}$ and $\pi^{0} \pi^{0}$ pairs for a very loose isolation criterion of $E_{T \max }=100 \mathrm{GeV}$ in a cone of $R=0.4$ at NLO. Note that the fully inclusive case would be plagued by uncertainties stemming from the low $-z$ regime of the pion fragmentation functions (see discussion below). To avoid this source of uncertainty we do not present figures showing the inclusive rate. The plot shows the importance of the reducible $\pi^{0} \gamma, \pi^{0} \pi^{0}$ backgrounds before isolation cuts. The $\pi^{0} \gamma$ component is more than an order of magnitude above the $\gamma \gamma$ background. The $\pi^{0} \pi^{0}$ background is of the order of several hundreds of $\mathrm{pb} / \mathrm{GeV}$. Tightening the isolation requirement by imposing $E_{T} \max =10 \mathrm{GeV}$ changes the production rate of these components. This is shown in Fig. 2. We remind that the very narrow Higgs signal will be a sharp peak - its width is determined by the detector resolution of around $1 \mathrm{GeV}$ - ranging from 50 to $100 \mathrm{fb} / \mathrm{GeV}$ in the given mass window [22]. The $\pi^{0} \pi^{0}$ background is reduced considerably whereas the $\gamma \pi^{0}$ background is still of the order of the irreducible diphoton background. Concerning the prompt photon pairs, the used isolation criterion reduces its rate by about a factor of two, because the fragmentation component of the prompt photon pair is vetoed by isolation cuts. Note that for the given isolation criteria not only direct photon pairs remain. For $E_{T \max }=10 \mathrm{GeV}$ almost 20 per cent of the total rate still come from fragmentation in the given plot for the scale choice used. Fig. 3 shows the suppression of the irreducible diphoton background when isolation is imposed. To suppress photons from fragmentation more efficiently

\footnotetext{
${ }^{1}$ In this version a bug was removed compared to the first version described in [7]. In that paper the main issue was the prediction of photon pairs. Only fragmentation contributions in the case of severe isolation cuts were affected by the bug. These are numerically irrelevant for the diphoton rate predictions.
} 
harder isolation cuts than the ones shown in the plot have to be imposed. A remaining uncertainty for the prompt photon pairs comes from higher order corrections to the box contribution. These corrections have been computed recently [23] but are not yet implemented in DIPHOX. To give an idea of the rejection factors due to isolation we have plotted the invariant mass distributions of the photon-pion pair for maximally allowed hadronic energies of $E_{T \text { max }}=100,20$, and $10 \mathrm{GeV}$ in a cone of $R=0.4$ in Fig. 4. Due to the collinear fragmentation model used the hadronic energy is mainly located along the fragmenting particle. This leads to the fact that there is no significant $R$ dependence present. Only genuine higher order $2 \rightarrow 3$ matrix elements can induce such a dependence and we only state that they are on the per cent level. In the case of photon-pion production two mechanisms for the production of the photon are possible. The photon can be produced directly in a hard interaction or through fragmentation. In the case of isolation the latter component is reduced by an order of magnitude as can be seen in Fig. 5. For completeness we provide with the plot for the invariant mass distribution of pion pairs, Fig. 6. In a realistic experimental situation the pion rates can be suppressed further. In the relevant $p_{T}$ range, an extra reduction factor of 2 to 3 per pion can be expected in fine-grained detectors with recognition of isolated $\pi^{0}$ as two overlapping showers $[24,25]$.

Another spectrum of relevance is the transverse momentum distribution of the pair produced particles, $q_{T}=\left|\vec{p}_{T 1}+\vec{p}_{T 2}\right|$. In Fig. 7 this distribution is shown for two isolation criteria. Recall that the full pion photon rate is built up out of the direct component and the fragmentation component. At colliders which operate at lower energies the fragmentation component is typically only a fraction of the full rate. Through isolation the fragmentation component dies out quickly. Fig. 8 gives an idea of the suppression factors due to isolation. Note that this theoretical curve is not reliable in the first few bins, as multiple soft gluon emission alters the divergent behaviour of the fixed order calculation. Near $q_{T}=0$ the partonic cross section diverges logarithmically. This divergence is diluted by convoluting the partonic cross section with the pion fragmentation function. Also at $q_{T}=E_{T \text { max }}$ there is a critical point inside the physical spectrum (see [7] for a discussion). The large bin width prevents from observing this singular behaviour. To deal with these issues in a rigorous way is beyond the scope of this study ${ }^{2}$.

Hard isolation cuts test fragmentation functions at high $z$ values, where current parametrisations are not at all constrained by experimental data. This problem is visualised in Fig. 9 where we show the photon-pion invariant mass distribution calculated with both, the KKP [15] and the older BKK [26] fragmentation functions. The KKP parametrisation leads to a higher prediction than the BKK one. The ratio of the two curves (KKP/BKK) is about 3/2. For dipion observables the situation is much worse. In Fig. 10 the discrepancy between the BKK and KKP parametrisations in the critical region is shown. One observes that at high $z$ values the fragmentation gluon-to-pion is strongly suppressed. Focusing on the quark-to-pion fragmentation, one sees that for high $z$ values the parametrisations differ considerably. This will be discussed in more detail below.

We have to comment now on the remaining theoretical uncertainty due to higher order corrections. As will be pointed out below the large $z$ region is dangerous for the predictive power of NLO calculations because terms are produced which are divergent as $z \rightarrow 1$. In Fig. 11, we plot LO and NLO predictions ${ }^{3}$ for the invariant mass distribution of the photon-pion pair for several isolation criteria. Note that the ratio of both curves is pretty stable with decreasing $E_{T \text { max }}$. The ratio of both curves lies around 1.7, a typical value for a NLO correction. We just mention that the situation worsens as $E_{T \text { max }}$ is reduced further which indicates the presence of large NLO contributions induced by pressing $z$ to one. As will be pointed out below there are two reasons for that. Phase space regions which are excluded at LO are populated at NLO and become dominant, and large logarithms of

\footnotetext{
${ }^{2}$ The corresponding resummations to be done are technically very involved and no satisfying procedure is defined yet.

${ }^{3} \mathrm{LO}$ refers here to the Born matrix elements whereas NLO parton densities and fragmentation functions are used.
} 
$(1-z)$ are introduced. To have a proper control on the latter fixed order effect, a resummation of the relevant contributions is needed. For the isolation criteria used in the given plots we conclude from the NLO to LO ratios that these effects are not yet dominant. Still, for harder isolation criteria the predictivity of our fixed order approach has to be critically investigated.

As the isolation cuts spoil compensations between scale dependent LO and NLO contributions the scale dependence remains important. To give an idea of the uncertainties we have plotted the variation of the invariant mass spectrum of $\pi^{0} \gamma$ pairs under the change of renormalization $(\mu)$, factorisation $(M)$ and fragmentation scale $\left(M_{f}\right)$ for a strict isolation criterion in Fig. 12. By multiplying our standard scale choice by $1 / 2$ and 2 one finds variation of about +40 per cent to -30 per cent. This variation is reduced if one uses less restrictive experimental cuts.

The importance of NLO calculations is best visible in distributions which are restricted by LO kinematics. No LO parton shower can provide for a correct description of events far away from the LO kinematics. A relevant observable to exemplify this point is the $q_{T}$ distribution of a photon-pion pair. Increasing the isolation criterion, suppresses the LO phase space more and more, such that finally the whole tail of the distribution is a pure NLO effect, see Fig. 13. For completeness we show the effect of isolation on this observable in Fig. 8. For a comparison of the shape of our DIPHOX prediction to PYTHIA see [27].

\section{Experimental cuts and higher order corrections}

To understand the reliability of the presented predictions it is necessary to discuss the interplay between experimental cuts and theoretical NLO results. QCD cross sections are stabilised by the inclusion of higher order corrections, as scale dependencies are reduced. This works generally the better the more inclusive the observables are. Yet, the collider experiments at the Tevatron and the forthcoming LHC do not perform inclusive photon measurements. The experimental selection of prompt photons requires isolation cuts. We always use the cone isolation criterion introduced in Eq. (2). For the hadronic energy propagating into the isolation cone two sources can be distinguished in an experimental setup. First there is a a component which stems from the actual partonic, hard reaction which may be mapped to certain Feynman diagrams - this component is calculable inside a QCD calculation to a given order. And second there is a component coming from multipleinteractions and other events in the same particle bunch (pile-up). One has to be aware of the fact that an experimentally used $E_{T \max }$ is thus always bigger than a partonic value used in our study. It is even so that the experimental values $E_{T \max }$ are so small that a large fraction of the allowed hadronic transverse energy accompanying the candidate photon may come from such events. This leaves not much phase space for partonic fragmentation reactions. In the following we mean with $E_{T \max }$ only the hadronic energy stemming from the hard interaction. The sensitivity on $E_{T \max }$ defined in Eq. (2) is best seen by looking at the fragmentation variable $z$, which is the momentum fraction of a hadron, $p_{T h}(h=\pi, \gamma)$, and its parent parton $p_{T}$. At LO the kinematical constraints on this variable are induced by the experimental cuts through the collinearity assumption in the fragmentation model, $p_{T}=z p_{T}+(1-z) p_{T}=p_{T h}+E_{T}^{h a d}$. One finds

$$
z>z_{\text {min }}=\frac{p_{T \min }}{p_{T \min }+E_{T \max }}
$$

where $p_{T \text { min }}$ is the experimental cut on $p_{T h}$. Evidently, the variable $z$ is pressed towards one, as the ratio $E_{T \max } / p_{T \text { min }}$ decreases, means for hard isolation. At NLO, where extra radiated partons are present, this bound is even more severe as the veto is more easily fulfilled. The bulk of the pions which are produced in collider events correspond to values of $z$ substantially smaller than 1 . Hard isolation tests only the very end of the $z$ spectrum. The question is, wether we can reliably estimate the production rate of these high- $z$ pions, and especially of hard $\gamma \pi^{0}$ and $\pi^{0} \pi^{0}$ pairs. We find three aspects important in this respect, which are discussed in more detail in the following. 


\subsection{Reliability of fragmentation functions}

The usual fixed order perturbative QCD calculations of hadro production - as e.g. the one coded in DIPHOX - rely on a model of independent collinear fragmentation through fragmentation functions of a parton of species $j$ (quark of any flavour, or gluon) into a pion, $D_{j \rightarrow \pi^{0}}\left(z, M_{f}^{2}\right), z$ is defined above. Let us first comment on the uncertainties at small $z$, where this treatment is questionable. In particular the fixed order calculations do not resum the $\ln (1 / z)$ arising order by order in perturbation theory, which become large in the small $z$ region. The latter are associated with coherence effects in the multiple emission of soft gluons, which control the inclusive production of small $z$ pions $[28,29]$. Therefore this usual framework is notoriously inadapted to account for the production of these small $z$ pions. Moreover the dominant $z$ values at colliders are smaller than for fixed targets, as a consequence of scaling violations. Therefore the erroneous treatment of the fragmentation process at small $z$ will plague the estimates of inclusive dipion observables at colliders. This is the reason why the fits of independent fragmentation functions $D_{i \rightarrow \pi^{0}}\left(z, M_{f}^{2}\right)$ to experimental data presented in the literature $[26,15]$, and which we use in this work, start above some minimum value for $z$, e.g. $z_{\min } \sim 0.1$. The independent fragmentation framework is expected to account correctly for the production of pions with larger $z$ values. The requirement of a loose isolation criterion such as, for example $E_{T \max } \leq 100 \mathrm{GeV}$ for pions with $p_{T \pi^{0}} \geq 25 \mathrm{GeV}$ imposes that $z \geq 0.2$ over the whole $p_{T \pi^{0}}$ range of interest. To avoid uncertainties due to small $z$ values, as was pointed out already, we do not provide estimates for inclusive observables, but only for observables corresponding to pions submitted to isolation cuts.

The behaviour near the end point, $z \rightarrow 1$, is responsible for most of the uncertainties that plague the present study. Indeed, the use of very tight isolation cuts such as those used by the collider experiments select the vicinity of the elastic boundary $z \sim 1$. For example, in the Run Ib of the Tevatron, for prompt photon measurements, CDF required $E_{T \max }=2 \mathrm{GeV}$ in a cone $R=0.7$. For $p_{T} \geq 15 \mathrm{GeV}$, this means $z_{\min }\left(p_{T}\right) \geq \sim 0.9$. The value $E_{T \max }=2 \mathrm{GeV}$ required by the CDF experiment includes also the deposition of hadronic transverse energy coming from underlying events, while the latter are ignored in our partonic calculation as noted above. Consequently, the actual effective value of $E_{T \max }$ which should be used in a partonic calculation should be even smaller, the effective $z_{\min }$ would be even larger. However the fragmentation functions $D_{j \rightarrow \pi^{0}}\left(z, M_{f}^{2}\right)$ are fitted to data corresponding essentially to $z \leq 0.7$, i.e. much below the relevant region for the present study. Moreover, the data used in $[26,15]$ concern hadronic final states in $e^{+} e^{-}$annihilation. The gluon-topion fragmentation function is poorly constrained at moderate and large $z$ by this process because the subprocesses involving gluons in $e^{+} e^{-}$annihilation appear at higher order in the perturbative expansion in powers of $\alpha_{s}$, while subprocesses involving outgoing gluons contribute at lowest order in hadronic collisions. Although the contributions from gluon fragmentation are not expected to dominate, large uncertainties on their magnitude may still affect quantitative predictions. In order to illustrate how poor the present-day control over the large $z$ region is, it is instructive to compare the extrapolations of the KKP and BKK sets of parton-to- $\pi$ fragmentation functions. The KKP set is the most recent one, and it is constrained by more data, especially the LEP1 data at the $Z$ peak, in the region $0.1 \leq z \leq 0.7$. These two sets use different analytic ansätze. These different ansätze do not differ very much in the $z$ range in which they are most constrained by the $e^{+} e^{-}$data of course, but their respective extrapolations turn out to differ substantially from each other above $z \geq 0.7$, especially between $z=0.9$ and 1 , as shown in Fig. 10. This is true for the gluon, but also, though to a lesser extend, for the $u$ and $d$ fragmentation functions. This translates into a large uncertainty for the predictions of the reducible Higgs boson background in the channel $H \rightarrow \gamma \gamma$ in the mass range 80-140 GeV. Clearly a determination of fragmentation above $z \geq 0.7$ from data sensitive to this range is required for any further accurate prediction. Such an experimental input could in principle be provided by data on inclusive $\pi^{0}$ production at fixed target energy $[11]^{4}$, as well as measurements

\footnotetext{
${ }^{4}$ In Ref. [30], the comparison between E706 data and BKK fragmentation functions reveals an overall normalisation discrepancy. However, as noticed in Ref. [11], this problem seems to be largely reduced by using the KKP set.
} 
of isolated pions by the collider experiments $\mathrm{CDF}$ and $\mathrm{D} 0$, in association with their prompt photon measurements.

It is worth mentioning that the present inaccuracy of the description of fragmentation at large $z$ is not only a problem confined to the type of perturbative QCD calculations which are discussed in this article. They also potentially affect the fragmentation stage of the "full hadronic event generators" such as PYTHIA [31] and HERWIG [32] as well. Indeed, the respective fragmentation ingredients of these event generators have been tuned to describe accurately the bulk of the data, especially at LEP, whereas the tails of the distributions near the end point have, to our knowledge, never been analysed in detail. In addition, the parton showers implemented in these event generators provide a effective summation of the soft gluon effects near $z \sim 1$ at a leading logarithmic accuracy; on the other hand they miss a part of the higher order corrections to the partonic hard subprocesses which may be large. The respective approximations in the "partonic calculations" on one hand, the full event generators on the other hand, being different, it would be instructive to compare the results given by both approaches [27].

\subsection{Large $z$ values and the need for end-point summation}

As the production of isolated pions at colliders and the one of inclusive pions at fixed target experiment, deal with $z$ values near the end point $z=1$, the theoretical calculation of the partonic subprocesses is confronted with the appearance of powers of large logarithmic terms $\ln (1-z)$ to any fixed order in perturbation theory. These large logarithms have in principle to be summed to all orders in order to improve - if not restore - the predictive power of the partonic calculation. The general framework to resum infrared logarithms in hadronic collisions has been settled in [33,34], however further work is needed to treat the endpoint summation in the case of one particle production, even more for pair production, where the kinematics is more constrained. In the present study, we investigate what can be done without such summations, relying on the extrapolations of the fragmentation functions available in the literature, and examining critically the limitations of our approach.

To be explicit let us consider the process $P P \rightarrow \gamma \pi^{0}+X$ in detail. The photon can be either direct or from fragmentation. The second case is considerably suppressed if one imposes severe isolation; thus we deal with the direct process only. At leading order there are two types of partonic processes: $q g \rightarrow q \gamma^{5}, q \bar{q} \rightarrow \gamma g$. At small $x$ values typical for the LHC the first is dominant. Radiative corrections give rise to initial state and final state singularities. Only the latter are of relevance for the point to be made here. They arise from the splitting of the outgoing parent quark into a quark and a gluon. Both may fragment into a pion but, again, for severe isolation one may focus on the dominant $q \rightarrow \pi^{0}$ fragmentation only. Thus we are looking at the case where a collinear gluon is radiated of a hard quark, indicated by the subscript $g / / q$, which subsequently fragments into a pion.

\footnotetext{
${ }^{5}$ Here $q$ stands for quark or antiquark.
} 
Following Ref. [7] the finite remnant of the collinear approximation of this correction is of the form

$$
\begin{aligned}
\left.\sigma(P P \rightarrow q g \gamma)\right|_{g / / q}= & \int d y_{\pi} d y_{\gamma} d p_{T \pi} \int_{x_{\text {min }}}^{1} \frac{d x}{x} \int_{z_{\text {min }}}^{1} \frac{d z}{z} \frac{\alpha_{s}(\mu)}{2 \pi} \\
& \times D_{\pi^{0} / q}\left(x, M_{f}^{2}\right) \frac{F_{q / P}\left(x_{1}, M^{2}\right)}{x_{1}} \frac{F_{g / P}\left(x_{2}, M^{2}\right)}{x_{2}}\left|\mathcal{M}_{q g \rightarrow q \gamma}^{L O}\right| \\
& \times\left[2\left(\frac{\ln (1-z)}{1-z}\right)_{+} a_{q q}^{(4)}(z)+\ln \left(\frac{p_{T \pi}^{2}}{x^{2} z^{2} M_{f}^{2}}\right) P_{q q}^{(4)}(z)+\ldots\right] \\
P_{q q}^{(4)}(z)= & C_{F} \frac{a_{q q}^{(4)}(z)}{(1-z)_{+}}+\frac{3}{2} C_{F} \delta(1-z) \\
a_{q q}^{(4)}(z)= & C_{F}\left(1+z^{2}\right)
\end{aligned}
$$

Here $y_{\pi}, p_{T \pi}$ are the rapidity and transverse momentum of the pion, $y_{\gamma}$ the rapidity of the photon, $x z$ the momentum fraction of the pion with respect to the parent quark, $p_{T \pi}=x z p_{T q}$, and $z$ the momentum fraction of the gluon with respect to the parent quark, $p_{T g}=z p_{T q}$. In the given collinear approximation the momentum fractions of the initial partons are

$$
\begin{aligned}
x_{1} & =\frac{p_{T q}+p_{T g}}{\sqrt{S}}\left[\exp \left(y_{\pi}\right)+\exp \left(y_{\gamma}\right)\right] \\
x_{2} & =\frac{p_{T q}+p_{T g}}{\sqrt{S}}\left[\exp \left(-y_{\pi}\right)+\exp \left(-y_{\gamma}\right)\right] \\
z_{\text {min }} & =x_{\text {min }} / x
\end{aligned}
$$

The value of $x_{\min }$ will be discussed below, as it is the sensitive variable to isolation cuts. The dots stand for terms which are less dominant as $z \rightarrow 1$. They are not necessarily small. By writing $a_{q q}^{(4)}(z) / z=C_{F}\left[(1-z)^{2} / z+2\right]$, one sees that the first term in the bracket only leads to non logarithmic corrections. Thus, approximating the bracket by 2 and using that for $z \rightarrow 1, \ln (z)=\mathcal{O}(1-z)$, the $z$ integration can be done explicitly and one finds

$$
\begin{aligned}
\left.\sigma(P P \rightarrow q g \gamma)\right|_{g / / q}= & \int d y_{\pi} d y_{\gamma} d p_{T \pi} \int_{x_{\min }}^{1} \frac{d x}{x} \frac{\alpha_{s}(\mu) C_{F}}{\pi} \\
& \times D_{\pi^{0} / q}\left(x, M_{f}^{2}\right) \frac{F_{q / P}\left(x_{1}, M^{2}\right)}{x_{1}} \frac{F_{g / P}\left(x_{2}, M^{2}\right)}{x_{2}}\left|\mathcal{M}_{q g \rightarrow q \gamma}^{L O}\right| \\
& \times\left[\ln \left(1-x_{\min } / x\right)^{2}+\ln \left(\frac{p_{T \pi}^{2}}{x^{2} M_{f}^{2}}\right) \ln \left(1-x_{m i n} / x\right)+\ldots\right]
\end{aligned}
$$

The important point is that the behaviour of these terms changes drastically as one goes from the inclusive case, where $x>x_{\min }=2 p_{T \pi} / \sqrt{S}$ is much smaller than one, to the case of severe isolation which press $x$ to values near one. Using the fact that the fragmentation function behaves as $D_{\pi^{0} / q}(x) \sim N(1-x)^{\beta}$ with $\beta \sim 1$, one can write

$$
\frac{D_{\pi^{0} / q}(x)}{x}=\frac{D_{\pi^{0} / q}\left(x_{m i n}\right)}{x_{\min }}\left(1-\frac{x-x_{\min }}{1-x_{\min }}\right)+\mathcal{O}\left(1-x_{m i n}, 1-\beta\right)
$$


and perform the integral over $x$. Focusing on the double logarithmic terms, one finds

$$
\begin{aligned}
\left.\sigma(P P \rightarrow q g \gamma)\right|_{g / / q}= & \int d y_{\pi} d y_{\gamma} d p_{T \pi} \frac{\alpha_{s}(\mu) C_{F}}{\pi} \\
& \times\left(1-x_{\min }\right) \frac{D_{\pi^{0} / q}\left(x_{\min }, M_{f}^{2}\right)}{x_{\min }} \frac{F_{q / P}\left(x_{1}, M^{2}\right)}{x_{1}} \frac{F_{g / P}\left(x_{2}, M^{2}\right)}{x_{2}}\left|\mathcal{M}_{q g \rightarrow q \gamma}^{L O}\right| \\
& \times \frac{1}{2}\left[\ln \left(1-x_{\text {min }}\right)^{2}+\ln \left(\frac{p_{T \pi}^{2}}{M_{f}^{2}}\right) \ln \left(1-x_{\text {min }}\right)+\ldots\right]
\end{aligned}
$$

the dots stand for single logarithmic terms and terms which are suppressed by an extra factor of $\left(1-x_{\text {min }}\right)$ and $(1-\beta)$. Note that outside the bracket, up to the factor $\alpha_{s}(\mu) C_{F} /(2 \pi)$, one has just the approximation for the leading order contribution. This means that the bracket itself times this factor could be taken as a good approximation for the higher order part of a $K$ factor $^{6}$.

$$
K=1+\frac{\alpha_{s}(\mu)}{\pi}\left[\ln \left(1-x_{m i n}\right)^{2}+\ln \left(\frac{p_{T \pi}^{2}}{M_{f}^{2}}\right) \ln \left(1-x_{m i n}\right)+\ldots\right]
$$

In here a factor of two is included from the second process with initial partons $\bar{q} g$. To get a feeling for the typical scales in the case of isolation one may try to choose $M_{f}$ such that the large logarithms compensate for a $p_{T \pi} \sim p_{T \text { min }}$. This can be achieved by a (optimal) scale choice

$$
M_{f}^{o p t}=p_{T \pi \min } \sqrt{\frac{E_{T \max }}{p_{T \min }+E_{T \max }}}
$$

Plugging in $p_{T \text { min }}=25 \mathrm{GeV}^{7}$ and $E_{T \max }=10 \mathrm{GeV}$ one finds $M_{f}^{\text {opt }} \sim 10 \mathrm{GeV}$. This motivated us to choose for $M_{f}$ in the figures presented not the canonical choice $M_{\pi \gamma} / 2$ but rather $M_{\pi \gamma} / 8$. Note that for a much higher scale choice the logarithms become constructive. For the upper choice of parameters and using $M_{f}=2 p_{T \text { min }}$ arbitrarily large $\mathrm{K}$ factors are induced in the limit $x_{\text {min }} \rightarrow 1$. The fact that the optimal scale in Eq. (9) goes to zero, as $E_{T \text { max }}$ goes to zero indicates that the cone isolation criterion is not infrared safe in this limit. In other words, if the experimental value of $E_{T \max }$ is already saturated by events from the hadronic environment such that there is no phase space left for the hard parton, fixed order calculations are not applicable.

It should be stressed that the whole discussion was neglecting constant terms and sub-leading logarithms. Thus it should only give a rough idea of the much more involved, complete situation. Using the same approximations the exercise can be worked through keeping also non-leading terms. The result is that one encounters in realistic situations additive contributions which are of the same size as the considered ones. All this deserves further investigations especially in the light of a possible resummation of the large logarithms and the role of these terms.

\subsection{Hard isolation and asymmetric $p_{T}$ cuts}

In LHC studies concerning Higgs boson search the used $p_{T}$ cuts are commonly asymmetric. We want to make the point here that this jeopardizes the predictive power of NLO calculations. The interplay between such asymmetric cuts and hard isolation criteria leads at LO to vetoed phase space regions which are filled by NLO processes only. The effect is visualised in Fig. 14, where the LO and NLO

\footnotetext{
${ }^{6}$ Note the way this $K$ factor depends on the experimental cuts applied and on the scale choice. This is a nice example of the fact that for non inclusive quantities the naive use of $K$ factors is not at all adequate to take into account higher order corrections.

${ }^{7}$ Due to the NLO kinematics $p_{T \gamma} \geq p_{T \pi}$. For asymmetric $p_{T}$ cuts the photon will always be the particle restricted by the larger $p_{T}$ cut.
} 
DIPHOX prediction for the dipion $p_{T}$ spectrum is plotted for symmetric, both $p_{T}>25 \mathrm{GeV}$, and asymmetric cuts, $p_{T 1}>40 \mathrm{GeV}, p_{T 2}>25 \mathrm{GeV}$. In the case of asymmetric cuts one observes that the LO component is suppressed for low $p_{T}$ values. This can be understood as follows:

Due to transverse momentum conservation, one has

$$
p_{T 1}+E_{T 1}^{h a d}=p_{T 2}+E_{T 2}^{h a d}
$$

where the pion 1 is assumed to have higher $p_{T}$ than pion 2 . This means for the transverse momentum of the pair, $q_{T}$ that

$$
0 \leq q_{T}=p_{T 1}-p_{T 2}=E_{T 2}^{h a d}-E_{T 1}^{h a d} \leq E_{T}^{\max }
$$

and because $p_{T 1}>p_{T 1 \min }(=40 \mathrm{GeV}$ in our case $)$

$$
p_{T 2} \geq p_{T 1}-E_{T}^{\max } \geq p_{T 1 \min }-E_{T}^{\max }
$$

This means that in the case of $p_{T 1 \text { min }}-E_{T}^{\max }>p_{T 2 \text { min }}$ one has a phase space restriction coming from the isolation criterion. Note that a saturation of the bound, $p_{T 2}=p_{T 1 \min }-E_{T}^{\max }$ is only possible if $E_{T 1}^{\text {had }} \rightarrow 0$, i.e. $z_{1} \rightarrow 1$. But in this limit the fragmentation function goes to zero and suppresses the cross section additionally. This will not happen in the case of photon-pion production where the same formulas apply with $E_{T 1}^{h a d}=0$. Eq. (12) is also true and leads to a restriction of the phase space but there is no additional suppression. The problem aggravates for lower $E_{T \max }$ values but becomes less relevant when $E_{T \max }>p_{T \min 1}-p_{T \min 2}$ and vanishes in the case of symmetric cuts $p_{T \min 1}=p_{T \min 2}$. The hole in the $p_{T}$ spectrum will be filled with events from higher order corrections. As the low $p_{T}$ region is energetically preferred, one gets numerically important NLO contribution to the cross section in the dipion case. The ratio between LO and NLO can become very big due to this effect. As pointed out, in photon-pion production the LO order contribution is less suppressed and the effect is milder. In the $q_{T}$ distribution shown in Fig. 8, one observes that only the first bin contains the LO contribution due to the same kinematical restriction mentioned above. On the other hand the NLO contribution is basically zero there (even below the plotted range). This is because pions with $p_{T}$ 's as large as the photon $p_{T}$ are disfavoured. The steep fall of the quark-to-pion fragmentation functions makes it very unlikely to find a photon-pion pair with $p_{T \gamma} \sim p_{T \pi}>40 \mathrm{GeV}$ which is needed to have $q_{T} \sim 0$.

From the discussion one sees that symmetric cuts are preferable from theoretical side for processes which have to be suppressed by isolation. Whereas this effect is for sure important for dipion production the effect is numerically sizable in the photon-pion case only for isolation criteria which are harder than the ones given in the presented figures. As can be inferred from Fig. 11, the ratio of the NLO to LO curves does not explode because the discussed phase space effect is not yet numerically sizable. Still we want to make the point that our NLO analysis suggest to choose symmetric transverse momentum cuts as theoretical higher order predictions tend to be more reliable in that case.

\section{Conclusions and outlook}

We have presented a full next to leading order study of the production of $\pi^{0} \gamma$ as well as $\pi^{0}$ pairs with a large invariant mass relying on the computer code DIPHOX. We have presented estimates for invariant mass and transverse momentum of the pair $\left(q_{T}\right)$ distributions taking into account realistic experimental cuts. The NLO corrections are large. Theoretical calculation improves the scale dependences in the case of inclusive observables. On the other hand, the results remain plagued by large uncertainties even in the NLO approximation, if realistic isolation criteria are applied. One source of large uncertainties is our poor present knowledge of the parton-to-pion fragmentation 
functions at large $z$. The estimates which we have presented here rely on extrapolations of analytical ansätze for these fragmentation functions for values of $z$ above 0.8 , i.e. outside the region where they were fitted to the data. The spreading of the extrapolations of different sets of parametrisations available in the literature for $z$ above 0.8 illustrates this fact. Experimental information may be provided by inclusive measurements of single pions and pions pairs at fixed targets, e.g. as performed by the E706 experiment [12], as well as by measurements of isolated pions at colliders, as could be performed by the CDF [5] and D0 [6] experiments.

Apart from this more experimental issue we pointed out that our next-to-leading order approach is still plagued by relatively large uncertainties due to phase space effects related to the different LO and NLO kinematics and also the presence of potentially large logarithms $\ln \left(1-x_{\text {min }}\right)$, if severe isolation cuts are imposed. Concerning the phase space effect, we discussed that symmetric $p_{T}$ cuts for the pions/photons are preferable from theoretical side. Otherwise NLO corrections are magnified in phase space regions vetoed at lowest order. Our predictions for $\gamma \pi^{0}$ production seems still reliable, up to scale variations of around 40 per cent, for the used isolation cuts. The same cannot be said for $\pi^{0} \pi^{0}$ production. The region $z \rightarrow 1$ is probed in a more critical way in this case. Further, predictions are very sensitive to the unconstrained part of fragmentation functions. We have to conclude that the uncertainties for this component of the reducible background for Higgs searches are not under control from theoretical side even at the next-to-leading order level. We only quote that we find large $\mathrm{K}$ factors and large scale variations, factors of 3 to 4 respectively, if we use the same isolation cuts as was done for the isolated $\gamma \pi^{0}$ production case. Fortunately this component to the diphoton background can be suppressed experimentally by separating pions from photons by distinguishing the shapes of the corresponding electromagnetic showers. Reduction factors of 2 to 3 per pion are possible and compensate somewhat the poor knowledge of the pion rates. Still, a more satisfactory theoretical knowledge of the dipion and pion/photon rates at LHC requires a better understanding of fragmentation functions near $z=1$, the resummations of large endpoint logarithms $(1-z)$, together with the use of experimental cuts which do not destabilise compensations between LO and higher order contributions.

\section{Acknowledgements}

We thank K. Lassila-Perini, V. Tisserand and E. Tournefier for discussions and comments. This work was supported in part by the EU Fourth Training Programme "Training and Mobility of Researchers", Network "Quantum Chromodynamics and the Deep Structure of Elementary Particles", contract FMRX-CT98-0194 (DG 12 - MIHT). LAPTH is a "Unité Mixte de Recherche (UMR 5108) du CNRS associée à l'Université de Savoie".

\section{References}

[1] ATLAS Technical proposal, CERN/LHCC 94-43; ATLAS detector and physics performance TDR, CERN/LHCC 99-14.

[2] CMS Technical proposal, CERN/LHCC 94-38; CMS ECAL TDR CERN/LHCC 97-33.

[3] The ATLAS and CMS Collaboration, ed. by J. G. Branson, D. Denegri, I. Hincliffe, F. Gianotti, F. E. Paige and P. Sphicas, preprint BNL-HET-01-33 - hep-ph/0110021.

[4] S. Catani et al., in Proc. CERN Workshop on Standard model physics (and more) at the LHC, Geneva 1999, ed. by G. Altarelli and M. Mangano, 1-113 CERN-TH-2000-131 - hep$\mathrm{ph} / 0005025$. 
[5] CDF Collaboration, F. Abe et al., Phys. Rev. Lett. 73 (1994) 2662; Erratum-ibid.74 (1995) 1891.

[6] D0 Collaboration, B. Abbott et al., Phys. Rev. Lett. 84 (2000) 2786.

[7] T. Binoth, J. P. Guillet, E. Pilon, M. Werlen, Eur. Phys. J. C16 (2000) 311.

[8] T. Binoth, Talk given at 35th Rencontres de Moriond on QCD and Hadronic Interactions, Les Arcs, France, March 2000, hep-ph/0005194.

[9] T. Binoth, J. P. Guillet, E. Pilon, M. Werlen, Report of the QCD/SM Working Group, Les Houches Workshop "Physics at TeV Colliders", 1999, Ed. by P. Aurenche et al. - hep$\mathrm{ph} / 0005114$.

[10] T. Binoth, J. P. Guillet, E. Pilon, M. Werlen, Phys. Rev. D63 (2001) 114016.

[11] T. Binoth, J. P. Guillet, E. Pilon, M. Werlen, hep-ph/0111043, to appear in Eur. Phys. J. C.

[12] E706 Collaboration, L. Apanasevich et al., Phys. Rev. Lett. 81 (1998) 2642;

M. Begel, Production of High Mass pairs of Direct Photons and Neutral Mesons in a Tevatron Fixed-Target Experiment, Ph.D. Thesis, Rochester University, NY, 1999 - hep-ex/9711017.

[13] T. Binoth, Talk given at 36th Rencontres de Moriond on QCD and Hadronic Interactions, Les Arcs, France, 17-24 March 2001, hep-ph/0105149.

[14] A. D. Martin, R. G. Roberts, W. J. Stirling, R. S. Thorne Eur.Phys.J.C4, (1998) 463.

[15] B. A. Kniehl, G. Kramer and B. Pötter, Nucl. Phys. B582 (2000) 514.

[16] S. Catani, J. P. Guillet, M. Fontannaz and E. Pilon, in preparation.

[17] H. Baer, J. Ohnemus and J. F. Owens, Phys. Rev. D42 (1990) 61.

[18] P. Aurenche, R. Baier and M. Fontannaz, Phys. Rev. D42 (1990) 1440.

[19] E. L. Berger and J. Qiu, Phys. Rev. D44 (1991) 2002.

[20] L. E. Gordon and W. Vogelsang, Phys. Rev. D50 (1994) 1901.

[21] W. Vogelsang and A. Vogt, Nucl.Phys. B453 (1995) 334.

[22] M. Spira, Fortsch. Phys. 46 (1998) 203.

[23] Z. Bern, A. De Freitas, L. J. Dixon, JHEP 0109:037,2001.

[24] ATLAS Calorimeter Performance TDR, CERN/LHCC 96-40

[25] CMS ECAL TDR CERN/LHCC 97-33

[26] J. Bienewiess, B. A. Kniehl and G. Kramer, Phys. Rev. D53 (1996) 6110.

[27] T. Binoth and K. Lasilla-Perini, to apear in the Report of the QCD/SM Working Group, Les Houches Workshop "Physics at TeV Colliders", 2001.

[28] A. Bassetto, M. Ciafaloni, G. Marchesini, Phys.Rep. 100 (1983) 201.

[29] Y. L. Dokshitzer, V. A. Khoze, A. H. Mueller, S. I. Troyan. Gif-sur-Yvette, France: Ed. Frontieres (1991) 
[30] P. Aurenche, M. Fontannaz, J. P. Guillet, B. Kniehl and M. Werlen, Eur. Phys. J. C13 (1999) 347.

[31] T. Sjostrand, P. Eden, C. Friberg, L. Lonnblad, G. Miu, S. Mrenna, E. Norrbin, Comput.Phys.Commun.135, (2001), 238.

[32] G. Corcella, I. G. Knowles, G. Marchesini, S. Moretti, K. Odagiri, P. Richardson, M. H. Seymour, B. R. Webber, JHEP 0101:010, 2001.

[33] R. Bonciani, S. Catani, M. Mangano, P. Nason, Nucl. Phys. B529 (1998) 424

[34] N. Kidonakis, G. Oderda and and G. Sterman, Nucl. Phys. B531 (1998) 365 


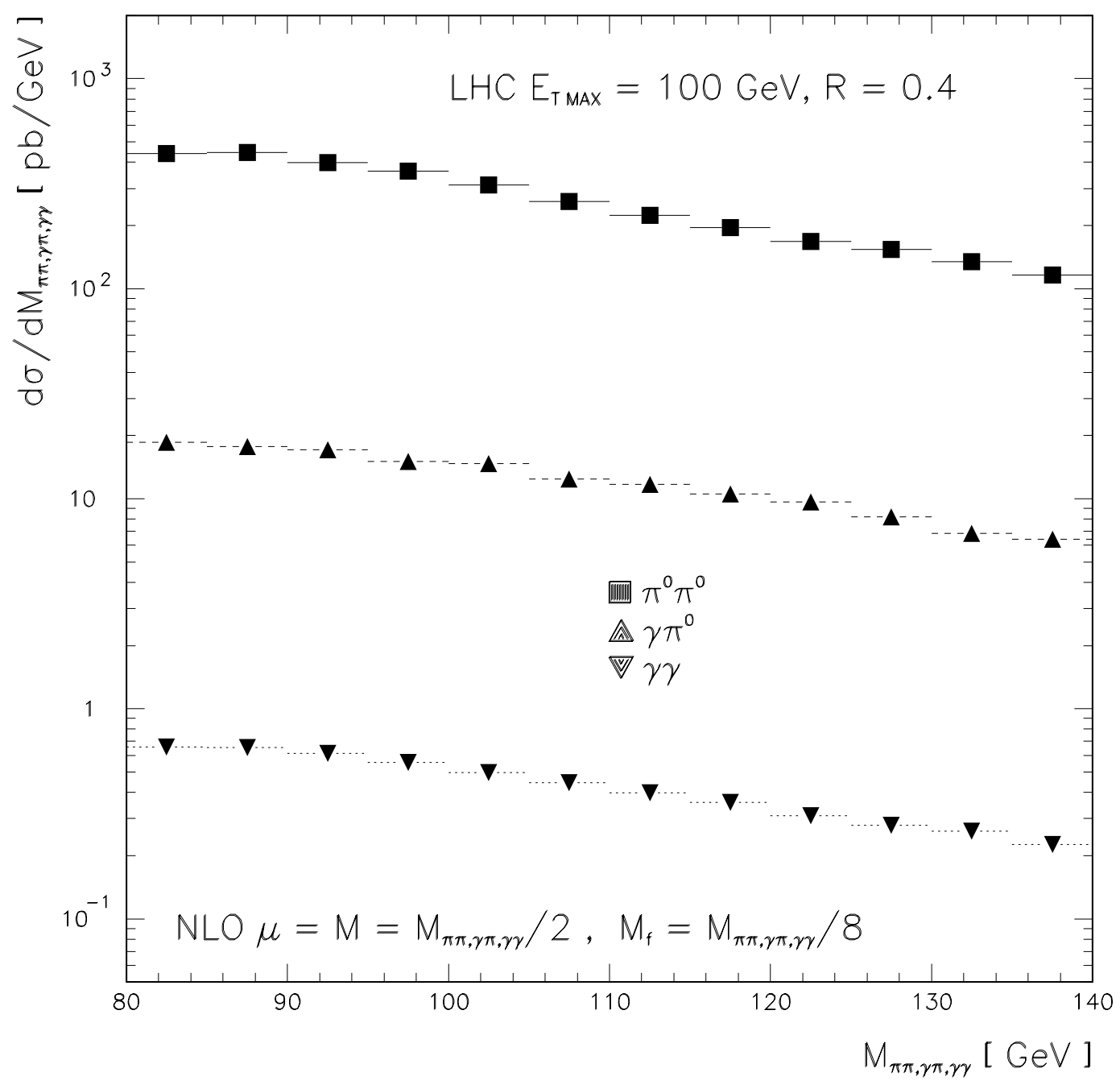

Figure 1: Comparison of the $\gamma \gamma, \gamma \pi^{0}$ and $\pi^{0} \pi^{0}$ invariant mass distribution for the isolation cuts $E_{T \max }=100 \mathrm{GeV}, R=0.4$. 


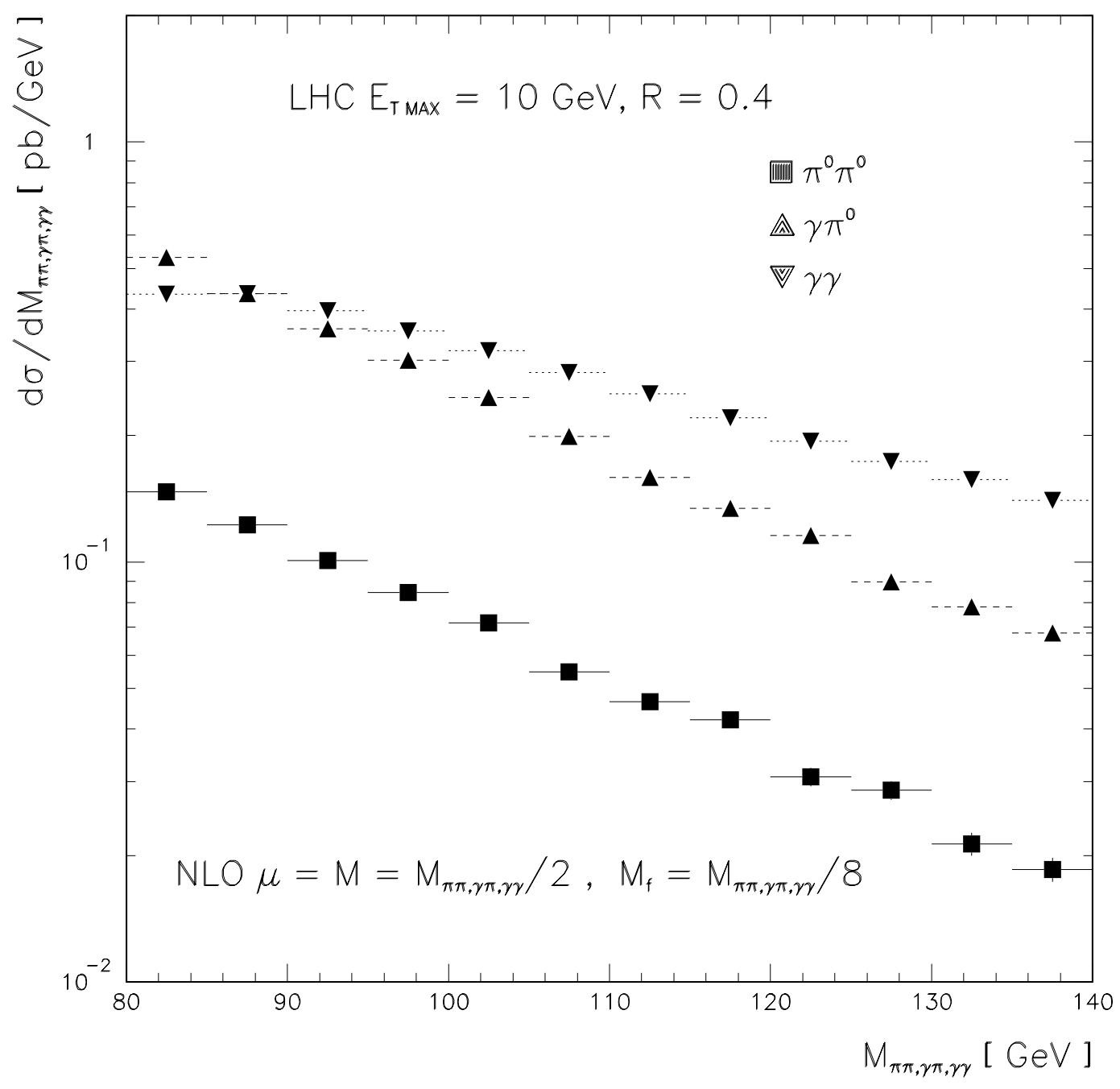

Figure 2: Comparison of the $\gamma \gamma, \gamma \pi^{0}$ and $\pi^{0} \pi^{0}$ invariant mass distribution for the isolation cuts $E_{\text {Tmax }}=10 \mathrm{GeV}, R=0.4$. 


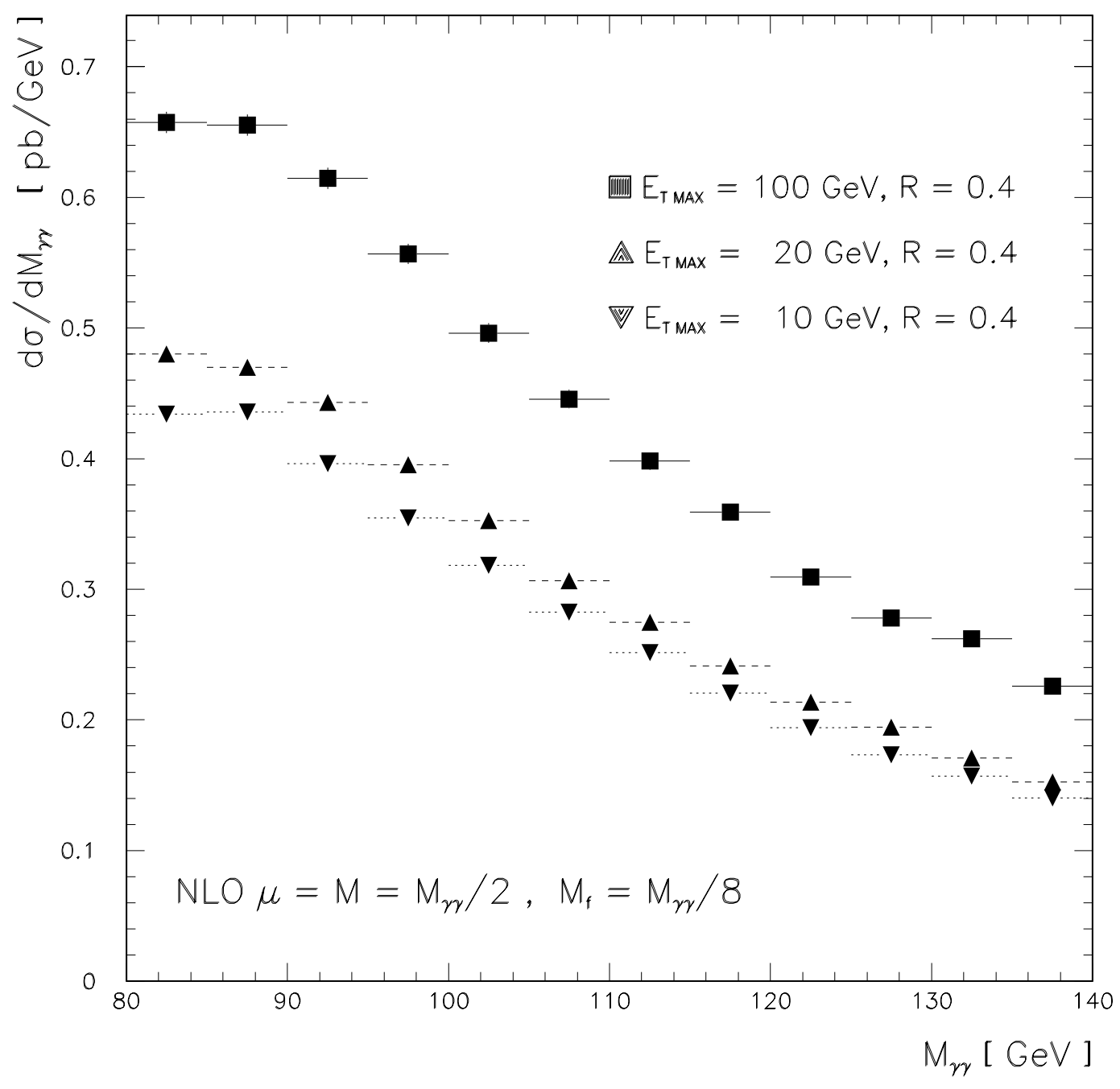

Figure 3: Invariant mass distribution of prompt photon pairs for various values of $E_{\text {Tmax }}$. 


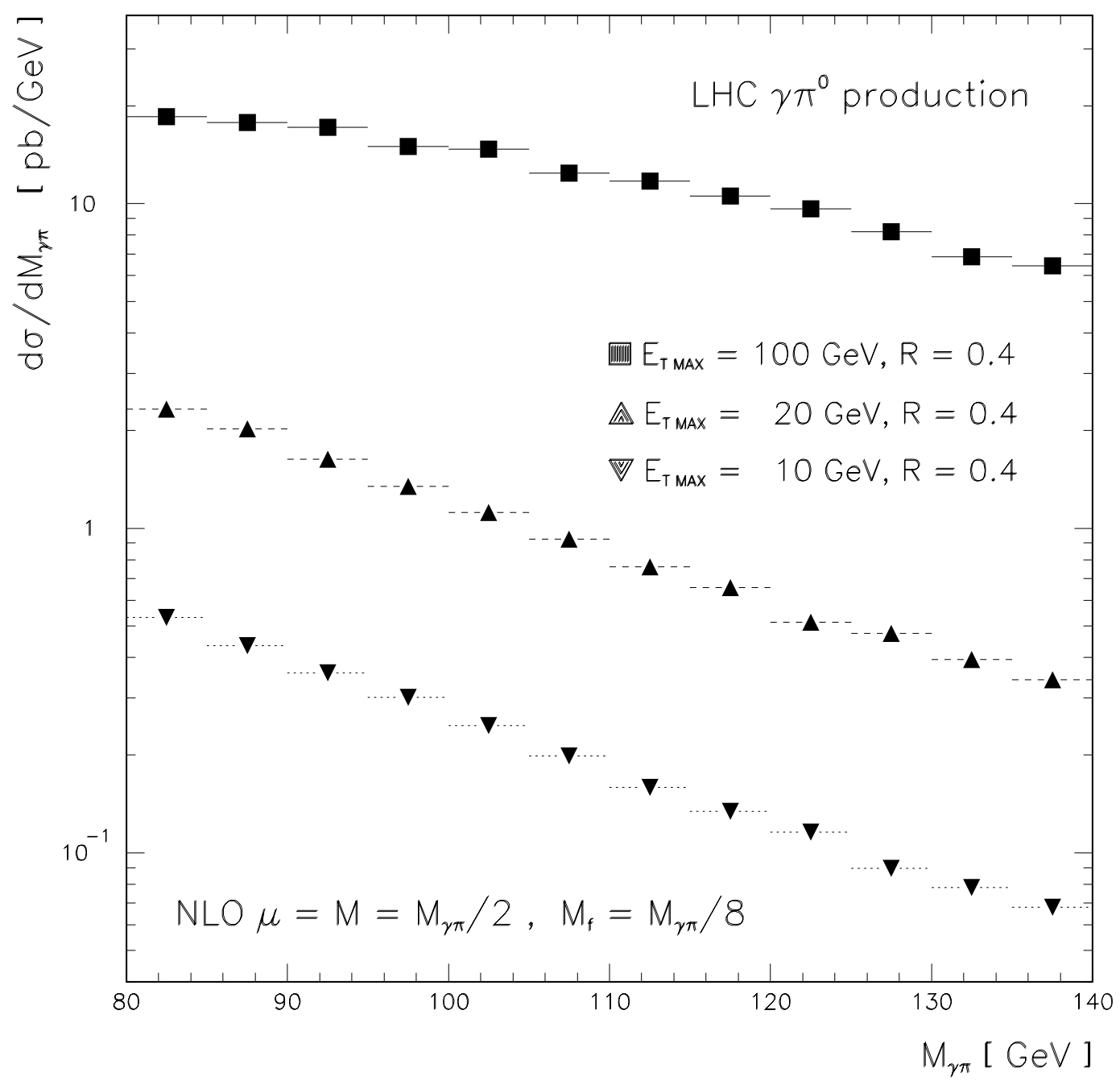

Figure 4: Invariant mass distribution of photon-pion pairs for various values of $E_{T \max }$. 

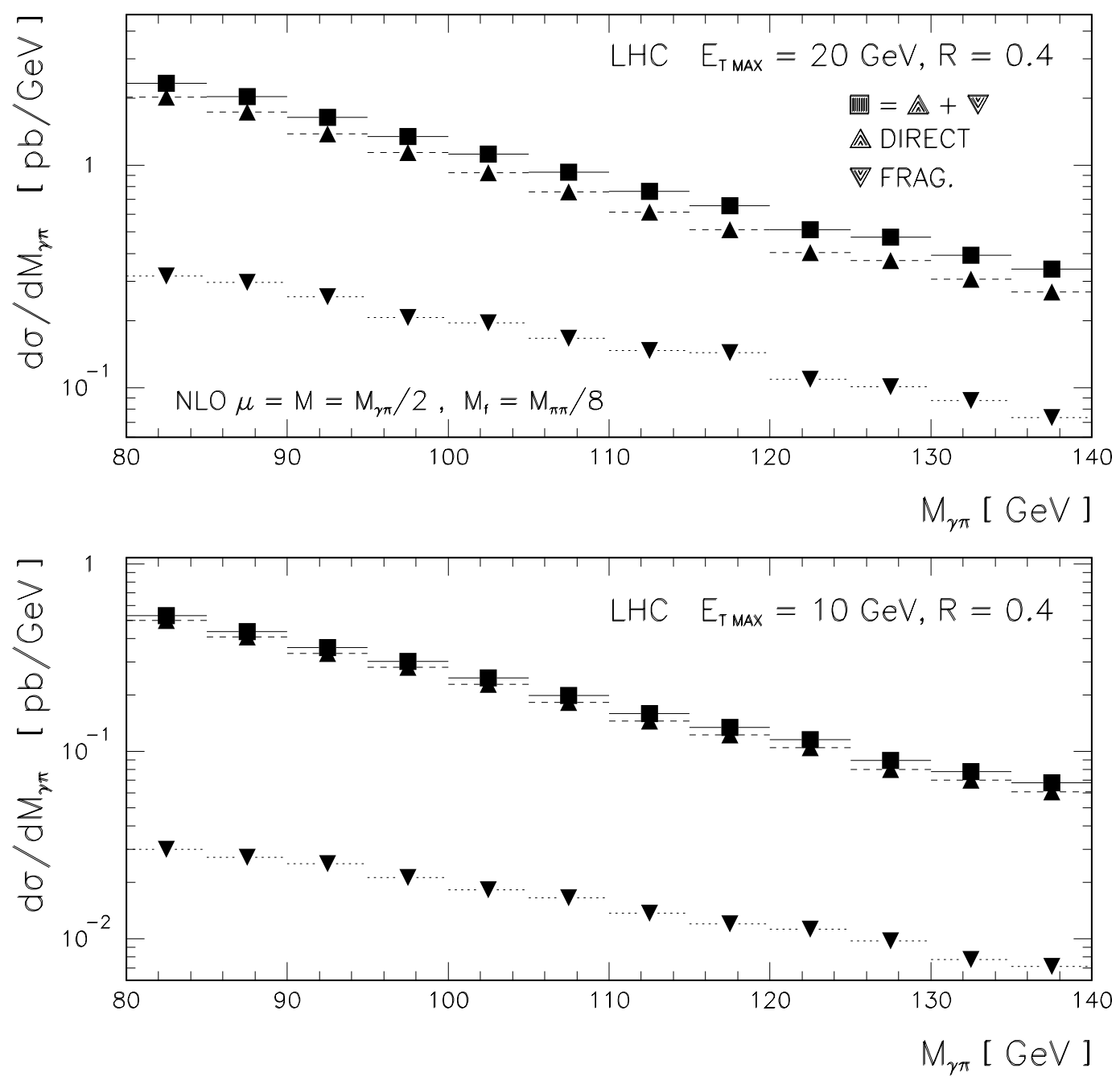

Figure 5: The suppression of the fragmentation component of $\pi^{0} \gamma$ production through isolation. 


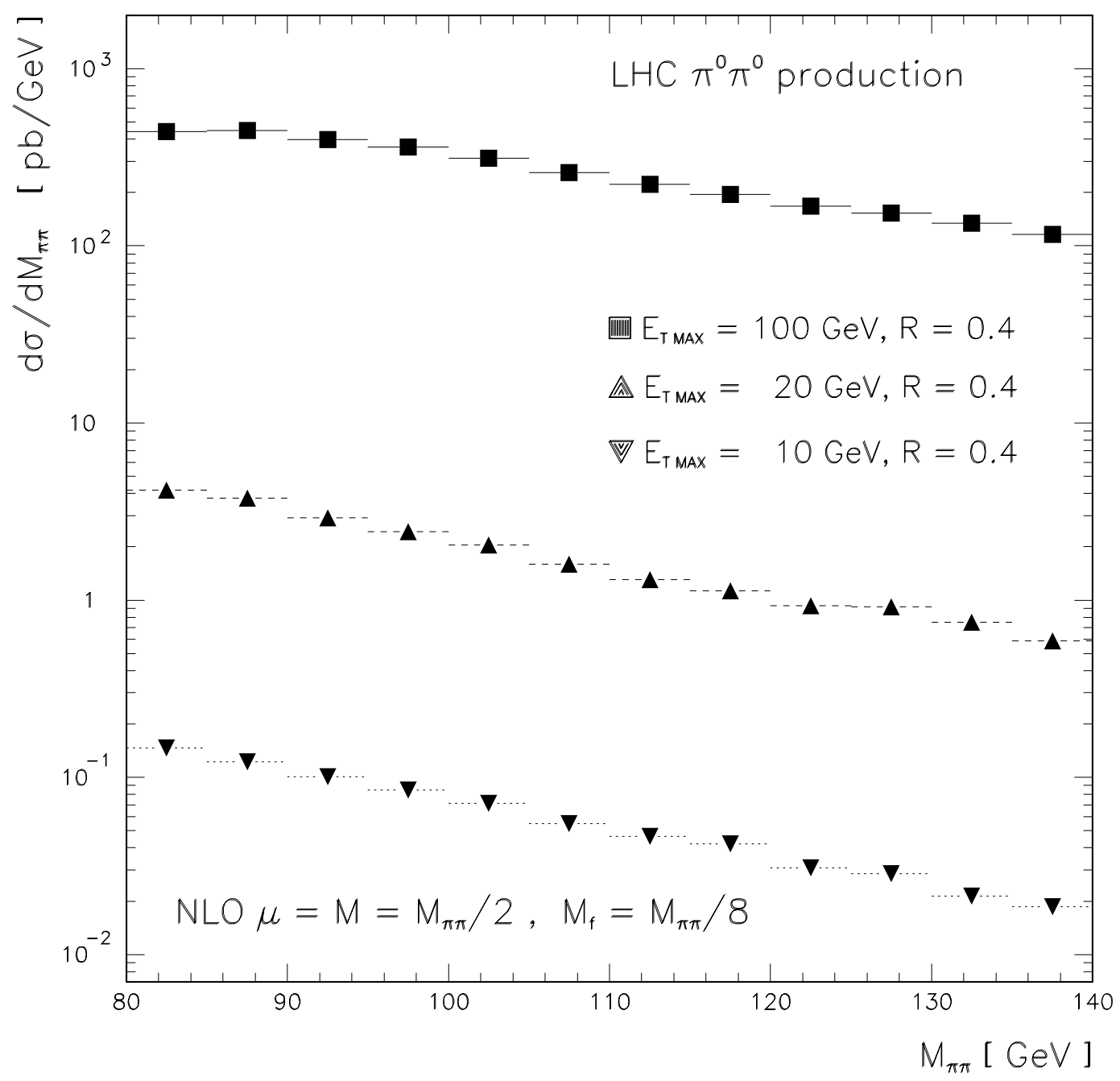

Figure 6: Invariant mass distribution of pion pairs for various values of $E_{\text {Tmax }}$. 

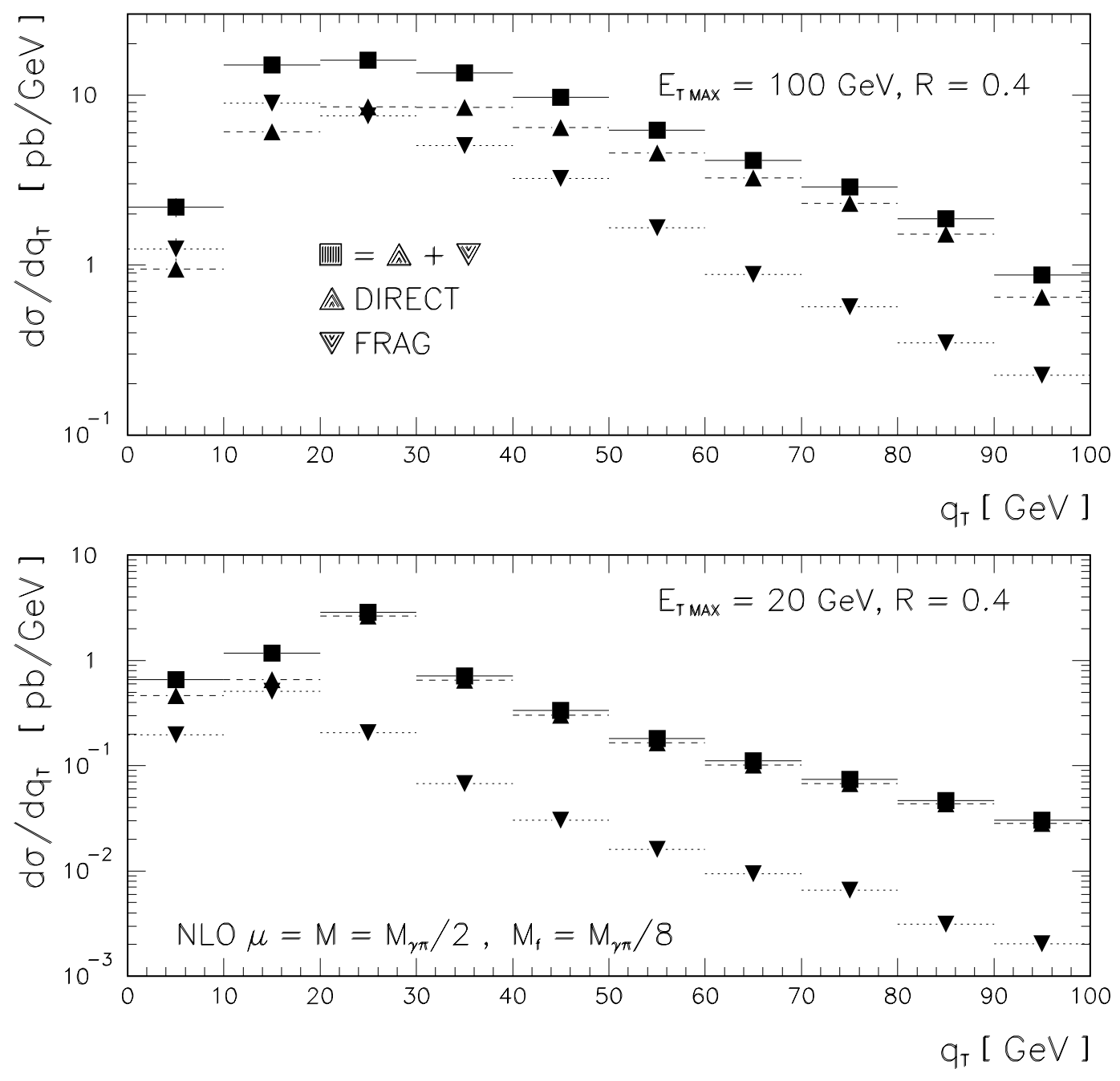

Figure 7: The $q_{T}$ dependence of photon-pion pairs split into direct and fragmentation part with (top) loose isolation and (bottom) severe isolation. 


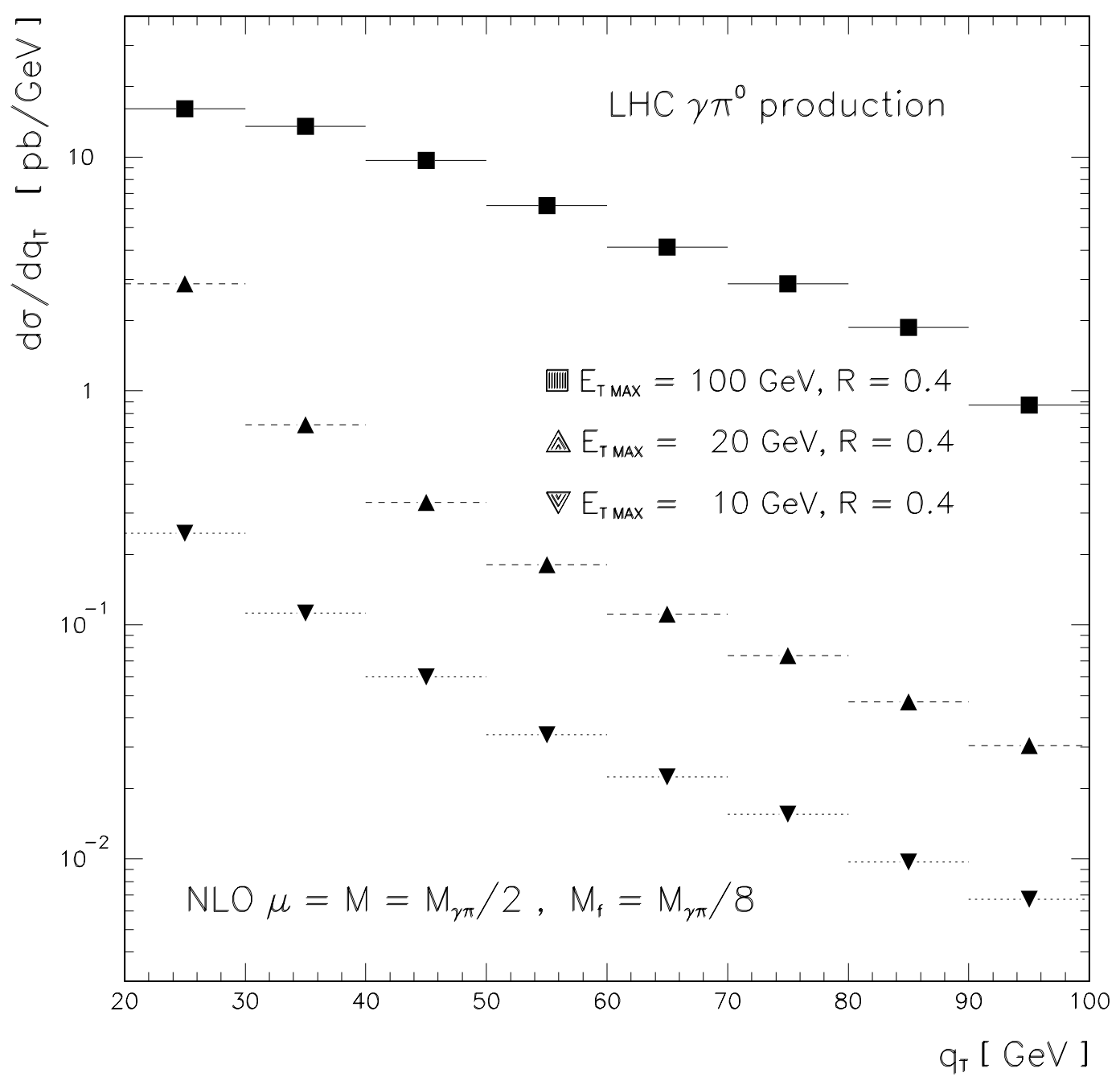

Figure 8: The $q_{T}$ dependence of photon-pion pairs for various isolation criteria. 


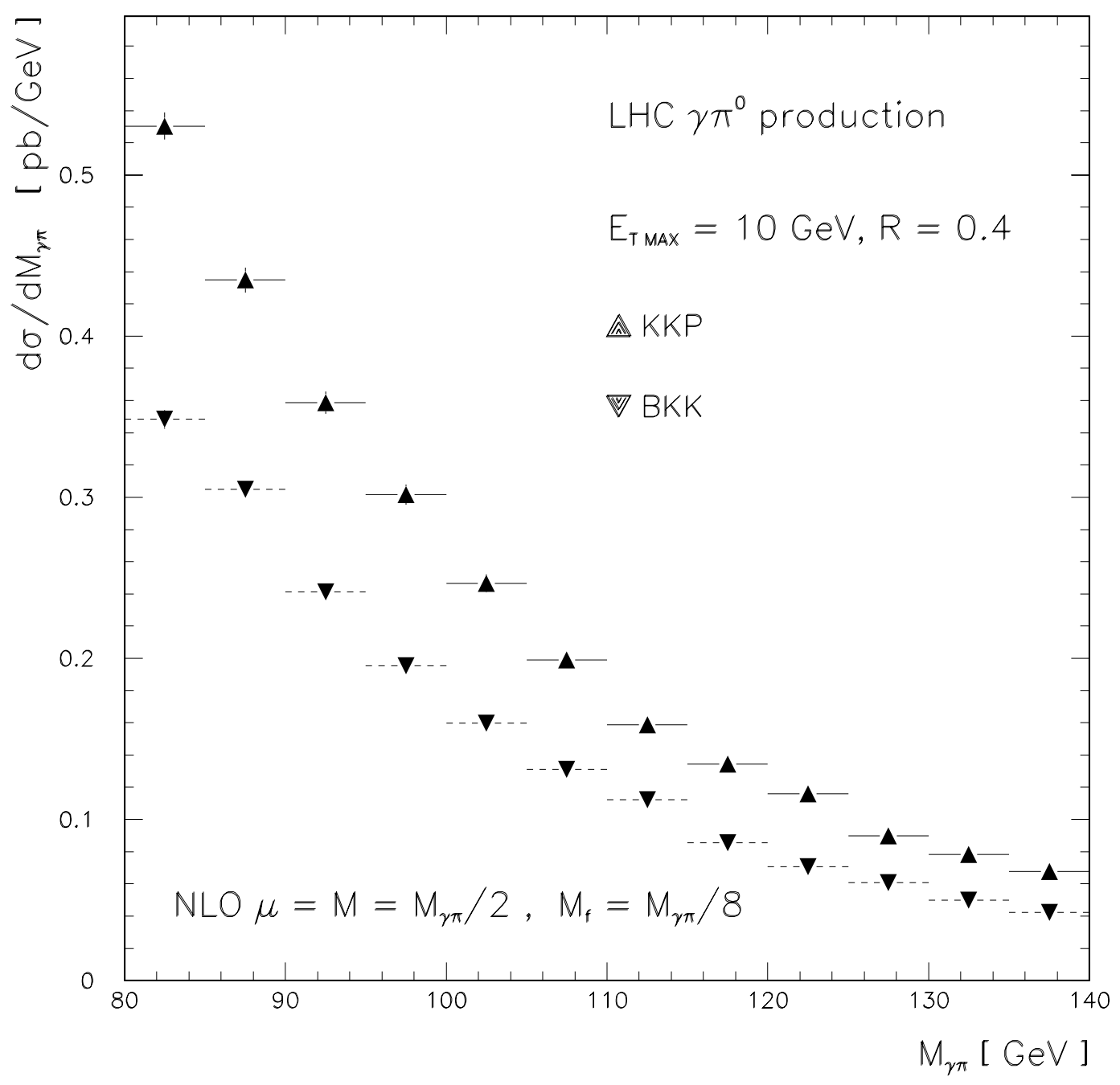

Figure 9: Comparison of the KKP and BKK fragmentation functions for $\pi^{0} \gamma$ production. 

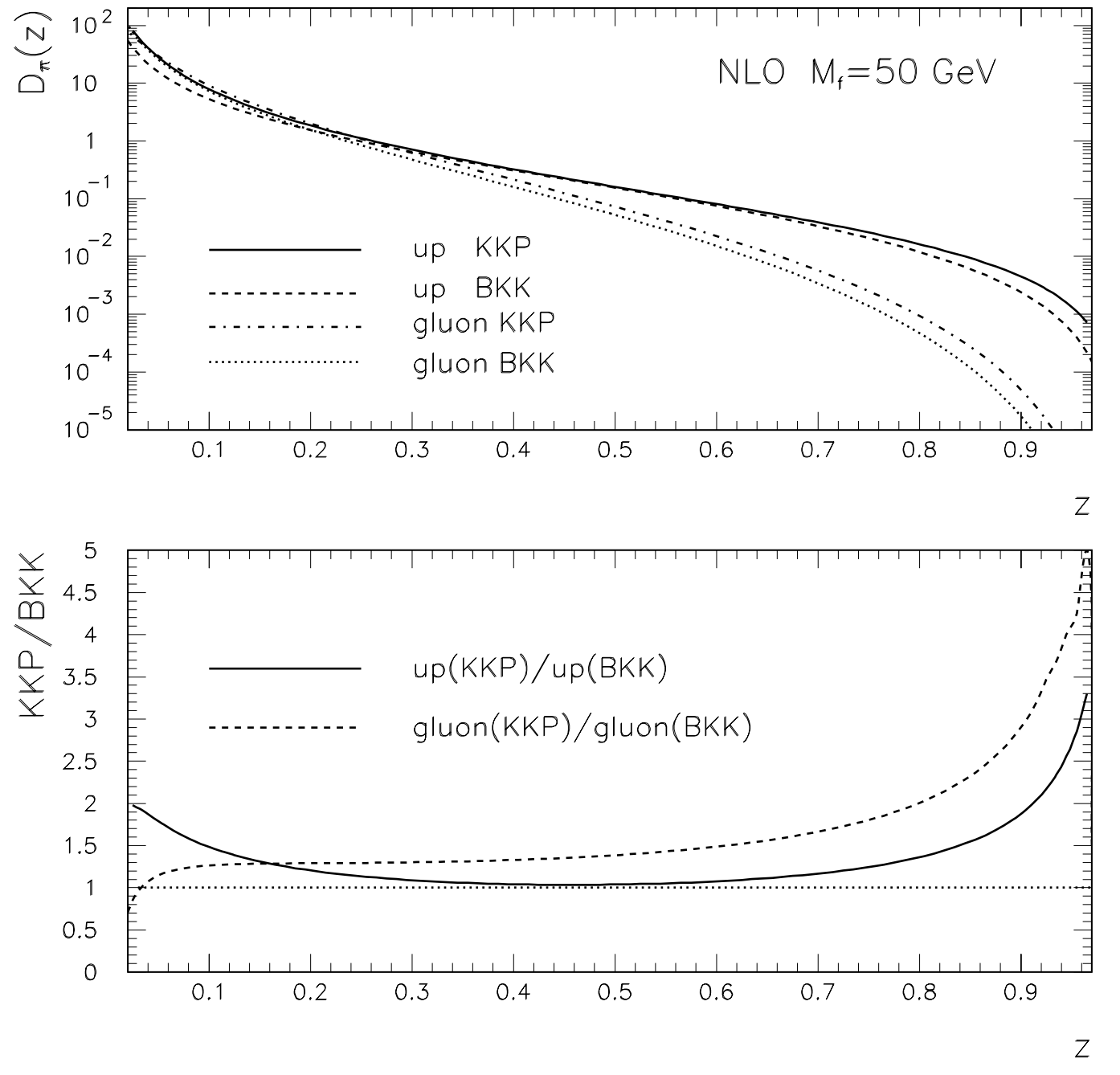

Figure 10: Comparison of KKP and BKK fragmentation functions for the fragmentation scale $M_{f}=$ $50 \mathrm{GeV}$ 

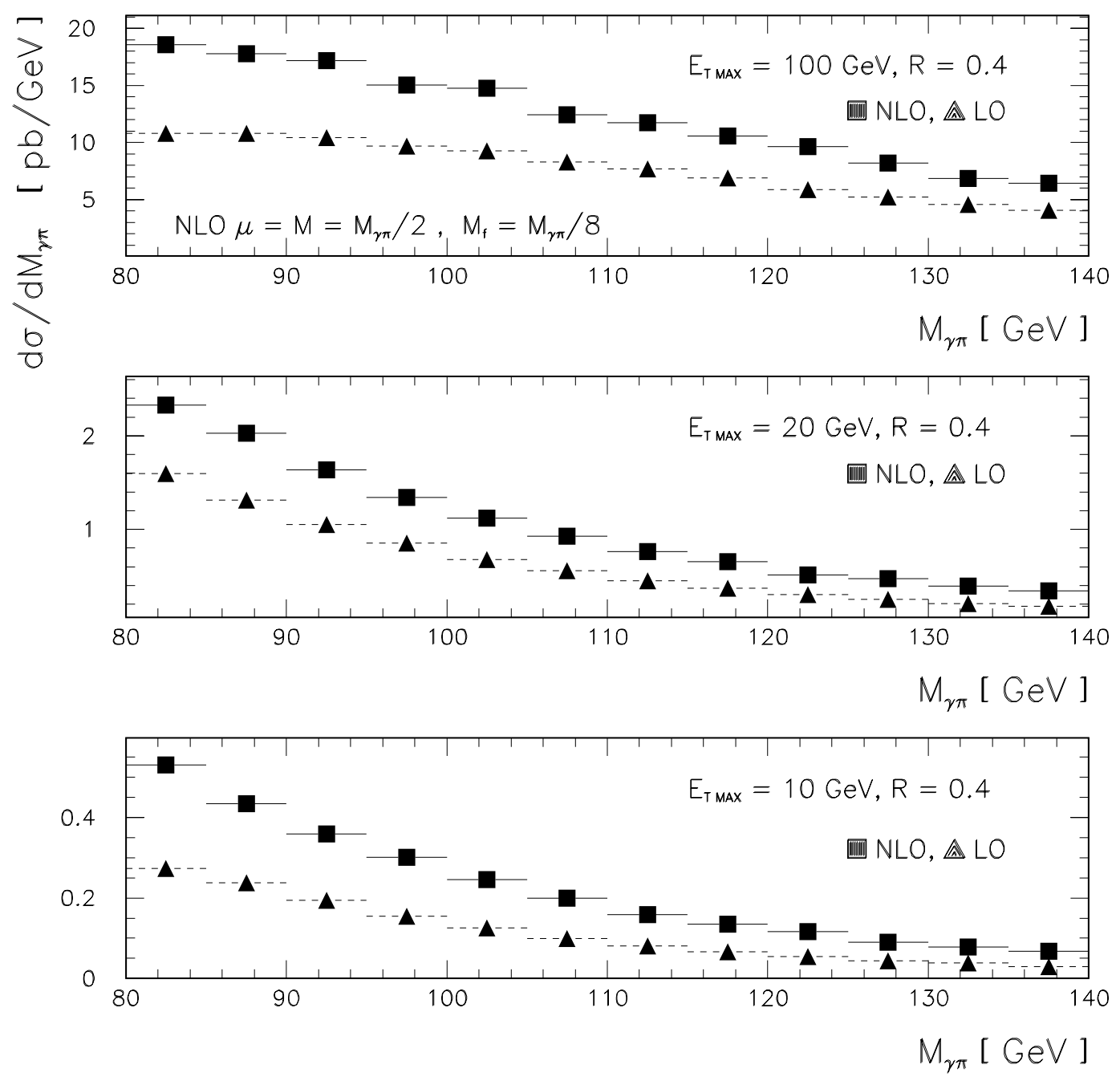

Figure 11: Comparison of $L O$ and NLO predictions of the invariant mass distribution of photon-pion pairs for various isolation criteria. 

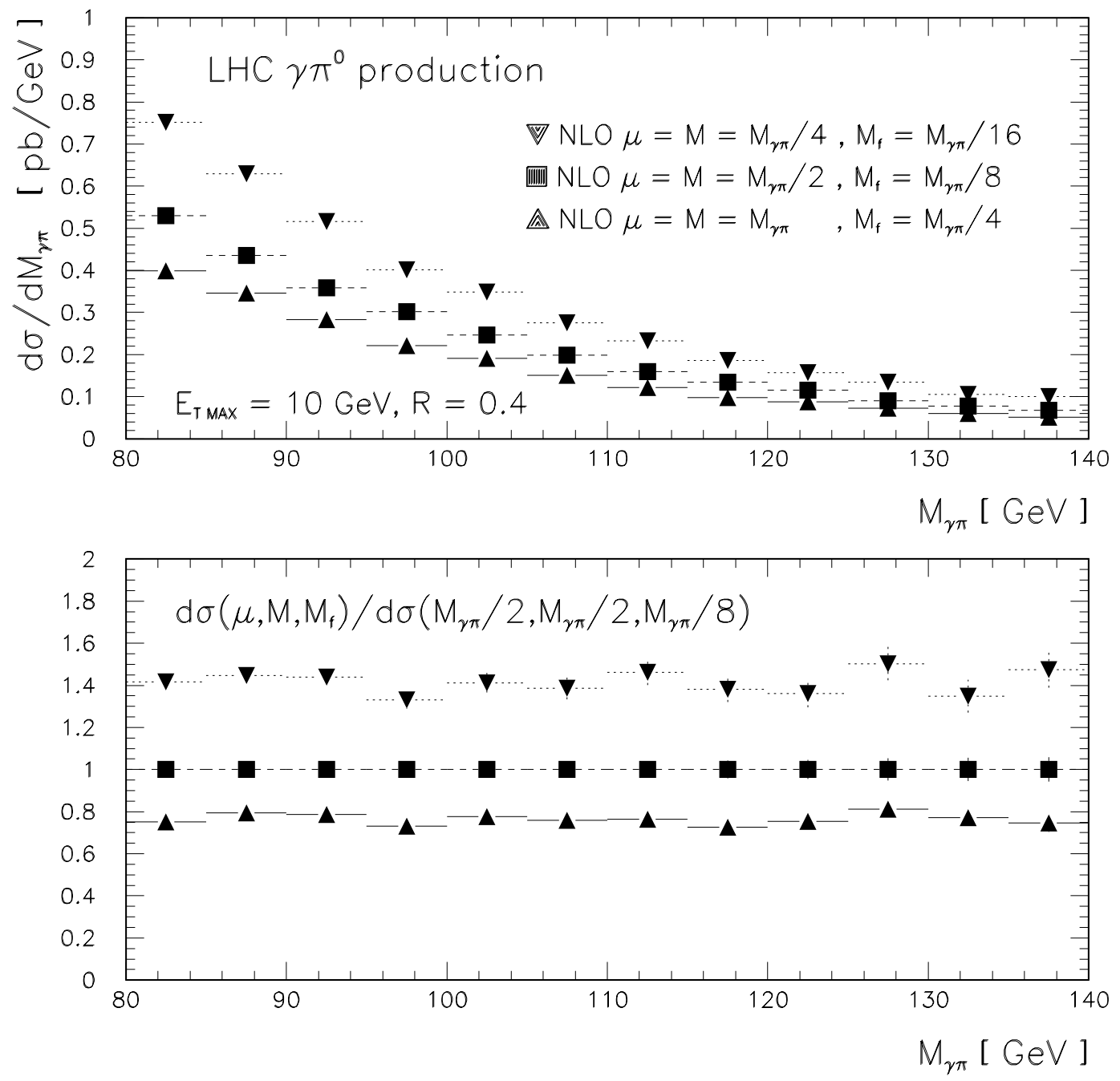

Figure 12: Scale dependence of the incvariant mass distribution of photon-pion pairs for the isolation criterion $E_{T \max }=10 \mathrm{GeV}$ in the cone $R=0.4$. The scales are varied around our standard choice $\mu=M=M_{\pi \gamma} / 2, M_{f}=M_{\pi \gamma} / 8$ (boxes), as explained in the text, by multiplying all scales by $1 / 2$ (inverse triangles) and 2 (triangles) respectively. 

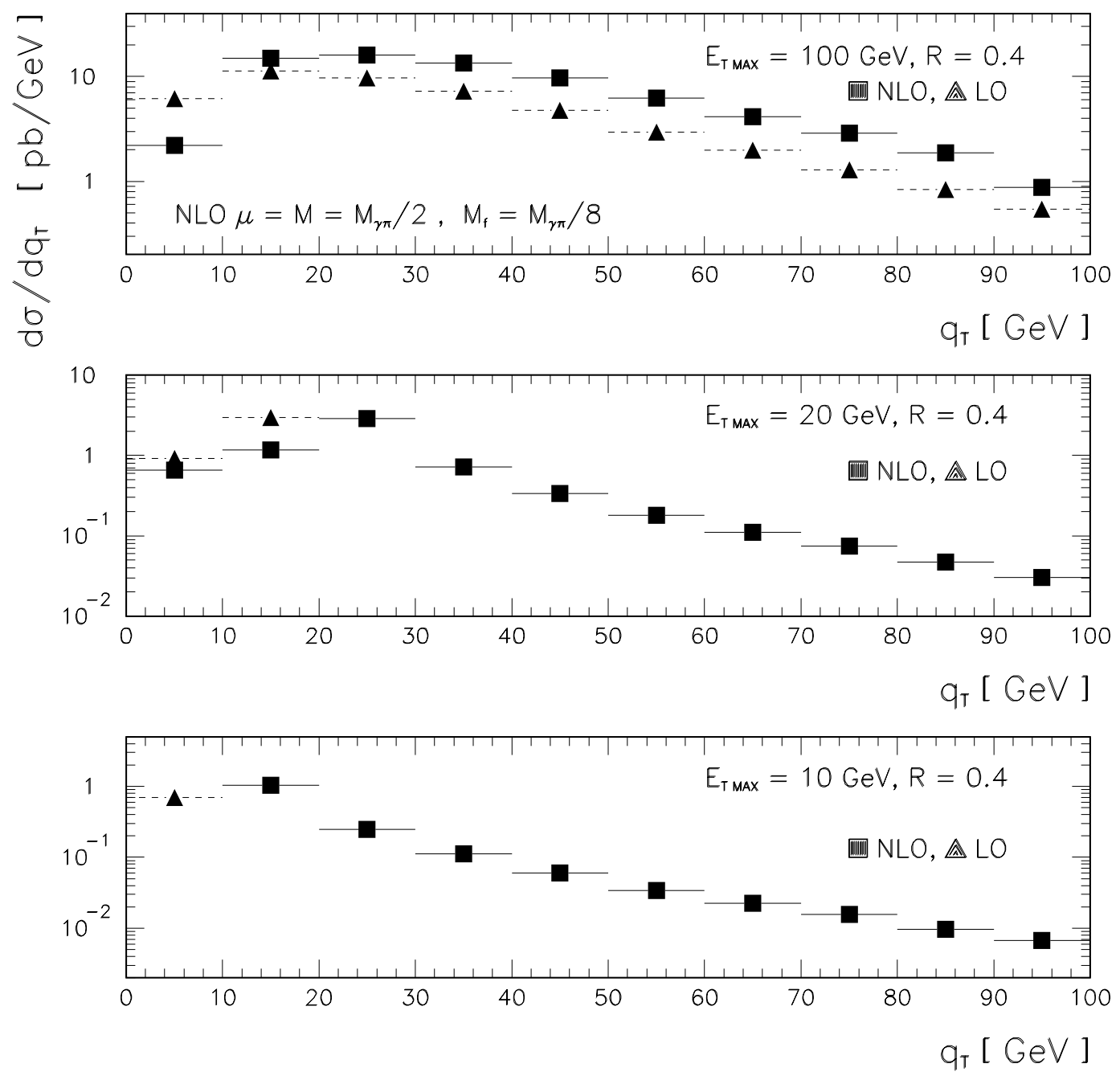

Figure 13: $L O$ and $N L O$ contributions for the $q_{T}$ distribution in $\pi^{0} \gamma$ production for different isolation criteria. The LO and NLO contributions populate different phase space regions when severe isolation is applied. 

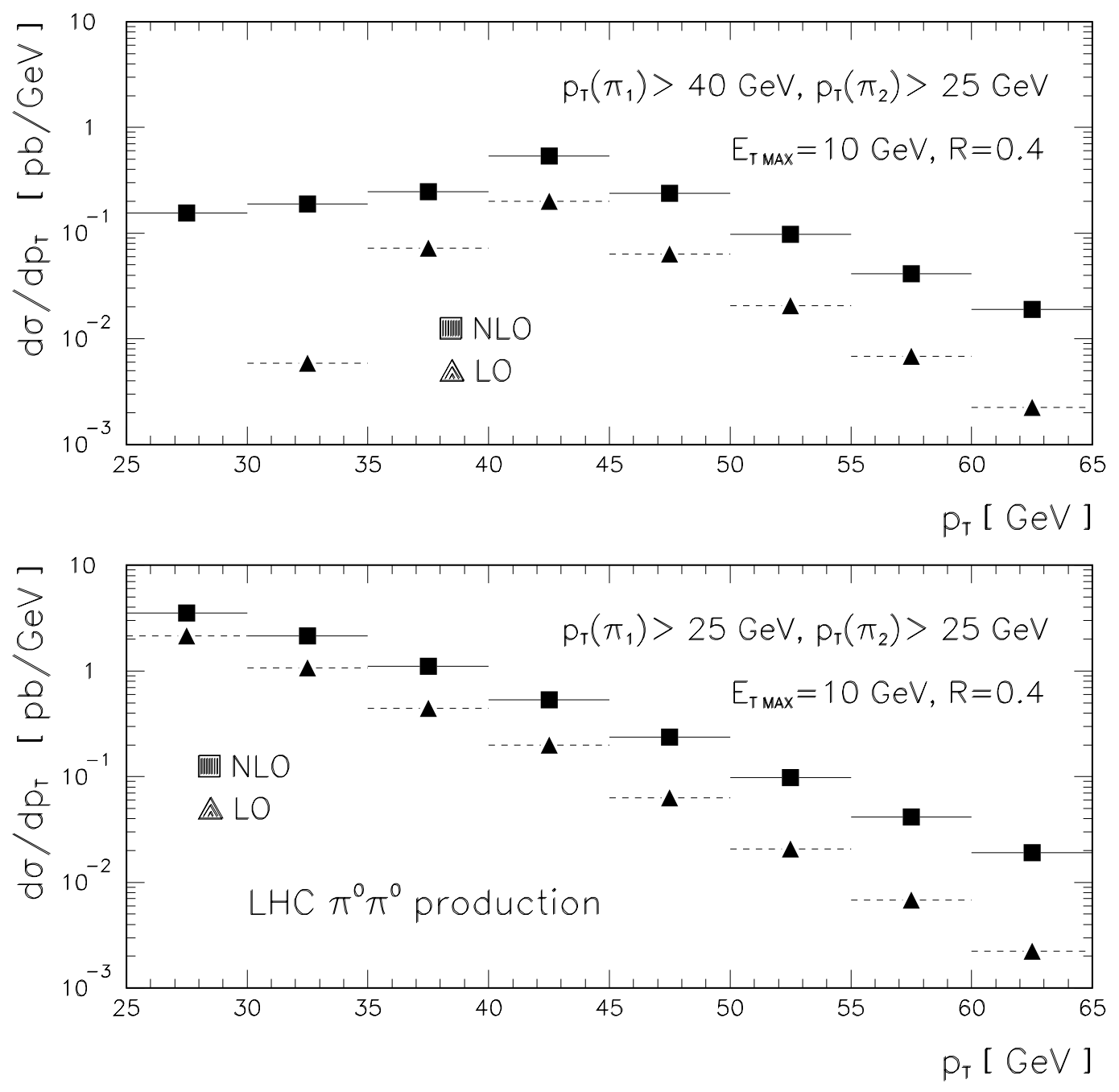

Figure 14: The transverse momentum spectrum of the pions in dipion production for symmetric (bottom) and asymmetric (top) $p_{T}$ cuts. Scales: $\mu=M=M_{\pi \gamma} / 2, M_{f}=M_{\pi \gamma} / 8$. 